\title{
A dhfr-ts- Leishmania major Knockout Mutant Cross-protects against Leishmania amazonensis
}

\author{
PST Veras ${ }^{+}{ }^{,}$, CI Brodskyn**, FMP Balestieri***, LAR de Freitas, APS Ramos, \\ ARP Queiroz**, A Barral**, SM Beverley****, M Barral-Netto*
}

\begin{abstract}
Laboratório de Patologia e Biologia Celular *Laboratório de Imuno-regulação e Microbiologia, Centro de Pesquisas Gonçalo Moniz-Fiocruz, Rua Valdemar Falcão 121, 40295-001 Salvador, BA, Brasil **Serviço de Imunologia, Hosp. Univ. Prof. Edgard Santos, UFBa, Salvador, BA, Brasil ***Laboratório de Tecnologia Farmacêutica, Departamento de Fisiologia e Patologia, UFPB, João Pessoa, PB, Brasil ****Department of Molecular Microbiology, Washington University School of Medicine, St. Louis, MO 63110, USA
\end{abstract}

E10-5A3 is a dhfr-ts- Leishmania major double knockout auxotrophic shown previously to induce substantial protection against virulent $\mathrm{L}$. major infection in both genetically susceptible and resistant mice. We investigated the capacity of $\mathrm{dhfr}^{-\mathrm{ts}^{-}}$to protect against heterologous infection by L. amazonensis. The degree of protection was evaluated by immunization of BALB/c or C57BL/6 mice with E10-5A3, followed by L. amazonensis challenge. Whether immunized by subcutaneous (SC) or intravenous (IV) inoculation, susceptible and resistant mice displayed a partial degree of protection against challenge with virulent $\mathrm{L}$. amazonensis. SC-immunized BALB/c mice developed lesions 40 to $65 \%$ smaller than non immunized mice, while IV immunization led to protection ranging from 40 to $75 \%$ in four out of six experiments compared to non immunized animals. The resistant C57BL/6 mice displayed comparable degrees of protection, 57\% by SC and 49\% by IV immunization. Results are encouraging as it has been previously difficult to obtain protection by SC vaccination against Leishmania, the preferred route for human immunization.

Key words: auxotrophic Leishmania major - Leishmania amazonensis - immunization - cross-protection

Leishmaniasis is an endemic disease in several parts of the World, including South and Central America, Africa and Asia. At least 400 million people are under the risk of infection, with an incidence of 600 thousand new cases of leishmaniasis per year and a prevalence of 12 million infected individuals (Desjeux 1992). People residing in endemic areas exhibit different clinical manifestations of leishmaniasis, determined by the species of infecting parasite and also by host factors.

Resistance and recovery to all forms of leishmaniasis has been linked to cell-mediated immune responses (Belosevic et al. 1989). Patients with New World cutaneous leishmaniasis (CL) and

This work was supported by grants from Fiocruz, Brazil, Pronex and the WHO Special Programme in Tropical Disease Research.

${ }^{+}$Corresponding author. Fax: 55-71-3564292. E-mail: pveras@svn.com.br.

CIB is a fellow of Capes/Fulbright Program and FMPB is a fellow of PICDT-Capes. PSTV, LARF, AB and MB-N are investigators of the Brazilian National Research Council (CNPq).

Received 26 October 1998

Accepted 2 March 1999 muco-cutaneous leishmanisis (ML) develop in vitro lymphocyte proliferative responses with abundant IFN- $\gamma$ (interferon- $\gamma$ ) production (Carvalho et al. 1985b), which may be induced by early IL-12 (interleukin 12) production. On the other hand, susceptibility is linked to a Th2 (T helper 2) type lymphocyte stimulation, with production of IL-4 (interleukin 4) (Bogdan et al. 1996, Romagnani 1996, Launois et al. 1997). Individuals with diffuse cutaneous leishmanisis (DCL) or visceral leishmaniasis (VL) mount a non-protective Th2 response (Carvalho et al. 1985a, Romagnani 1996).

For many reasons, it is important to develop a vaccine against leishmaniasis, and several lines of evidence suggest that this should be possible (Modabber 1990, Grimaldi Jr 1995, Handman 1997). Indigenous residents of highly endemic zones of zoonotic CL usually suffer the disease only once, and its severity is less than that occurring in non-indigenous visitors. Individuals from endemic areas have protected themselves from multiple lesions on the face by infecting their arms with virulent parasites, a practice known as leishmanization. Live vaccination has been used previously in several countries. However, since virulent organisms were used, vaccination-acquired leishmaniasis frequently proved more troublesome than the natu- 
rally-acquired disease. In addition, Leishmania is an easy parasite to manipulate and maintain in axenic culture. Several studies using experimental animals showed a protective effect in response to vaccination (reviewed in Barral-Netto \& Barral 1987, Modabber 1990, Connel et al. 1993).

The protection obtained by using live parasites has led to a search for attenuated parasites for immunization against leishmaniasis (Handman et al. 1983, Mitchell et al. 1984, Marchand et al. 1987, Kimsey et al. 1993) as the use of attenuated parasites could induce protection without producing pathology. Although the defect(s) of these lines is unknown, a defined auxotrophic line has been produced by targeted deletion of an essential metabolic gene, DHFR-TS (encoding the bifunctional dihydrofolate reductase-thymidylate synthase) in L. major (Cruz \& Beverley 1990, Cruz et al. 1991). This line did not cause disease even in highly susceptible mouse strains, and vaccination conferred substantial and specific protective immunity in $\mathrm{BALB} / \mathrm{c}, \mathrm{C} 57 \mathrm{BL} / 6$ and CBA mice challenged one week later with virulent L. major (Titus et al. 1995).

Studies with cross-protection are very rare (Alexander \& Phillips 1978, Howard et al. 1981, 1982), but it has already been demonstrated protection of BALB/c mice infected with L. tropica, a causative agent of Old World CL, against New World CL agents (L. panamensis, L. mexicana and L. amazonensis). In addition works of cross-protection using primates are controversial (Lainson \& Shaw 1977, Barral-Netto \& Barral 1987). In the present report, we evaluated the ability of the L. major dhfr-ts- mutant E10-5A3 to confer protection of mice against heterologous infection by L. amazonensis, a parasite which induces several different forms of leishmaniasis in humans (Barral et al. 1991).

\section{MATERIALS AND METHODS}

Animals - Inbred BALB/c and C57BL/6 were obtained from the Central Animal Facilities of Fiocruz (Rio de Janeiro, BR) and used beginning at eight weeks of age. Mice were maintained at Centro de Pesquisas Gonçalo Moniz (CPqGM), with water and balanced commercial ration ad libitum.

Parasites - The dhfr-ts ${ }^{-}$L. major (strain E105A3) (Cruz et al. 1991) was maintained in M199 medium, supplemented with $10 \mu \mathrm{g} / \mathrm{ml}$ Thymidine, $10 \mathrm{mM}$ Adenine, $0.25 \%$ Hemin, $20 \mathrm{mM}$ HEPES and 20\% Fetal Calf Serum (FCS, Hyclone). $L$. amazonensis, strain MHOM/BR-88/BA-125, was maintained in Schneider's medium supplemented with $10 \%$ FCS. $d h f r-t s^{\prime}$ and L. amazonensis stationary phase promastigotes were washed and suspended in saline for animal inoculation.
Immunization and challenge - Groups of susceptible mice $(\mathrm{n}=5)$ were immunized subcutaneously in the right footpad, or intravenously, with dhfr-ts $s^{-}$L. major suspension in saline containing $10^{4}, 10^{6}$ or $10^{8}$ E10-5A3 promastigotes in $20 \mu \mathrm{l}$ for SC or $200 \mu \mathrm{l}$ for IV vaccination and challenged one week later with $10^{6}$ stationary phase $L$. amazonensis promastigotes in the right footpad. The control group $(n=5)$ received an equal volume of phosphate-buffered saline (PBS). As previously described (Titus et al. 1995), mice were challenged one week later by SC inoculation in the right hind footpad with stationary phase $L$. amazonensis promastigotes $\left(10^{6}\right.$ or $5 \times 10^{6}$ parasites in $20 \mu \mathrm{l})$. The course of infection was monitored by measuring the footpad thickness using a dial gauge caliper. Graphs show the lesion size, expressed as the difference between the infected and the contralateral uninfected footpads. Text results are expressed as percentage of protection or the relative reduction of lesion size, compared to lesion from control, non-immunized mice.

\section{RESULTS}

We asked whether the $d h f r-t s^{-}$strain of $L . m a-$ jor was able to protect against heterologous challenge with promastigotes of $L$. amazonensis. We used the same protocol of immunization with which we previously obtained high degree of specific protection against homologous $L$. major challenge infection. Groups of susceptible BALB/c mice $(\mathrm{n}=5)$ were immunized subcutaneously with $10^{4}$, $10^{6}$ or $10^{8} \mathrm{E} 10-5 \mathrm{~A} 3$ promastigotes, and challenged one week later with $10^{6}$ stationary phase $L$. amazonensis promastigotes in the right footpad. Control animals received the same volume of saline. The group immunized with $10^{8}$ dhfr-ts- parasites displayed smaller lesions compared to the nonimmunized animals $(\mathrm{p}<0.001)$ or the groups immunized with $10^{4}(\mathrm{p}<0.01)$ or $10^{6}(\mathrm{p}<0.05)$ E105A3 (Fig. 1a). In four experiments, SC immunization conferred better protection between the 7 th and the 9th weeks post challenge infection. Protection ranged between 40 and $65 \%$. Overall, the degree of protection found was somewhat less than that observed previously by homologous challenge with virulent $L$. major (Titus et al. 1995).

Intravenous immunization of BALB/c mice was also evaluated. Animals were immunized with the doses of $10^{4}, 10^{6}$ or $10^{8}$ E10-5A 3 stationary phase promastigotes each. One week later, the immunized mice were challenged with $5 \times 10^{6} \mathrm{~L}$. amazonensis stationary phase promastigotes, 5 -fold more parasites used to challenge animals immunized by SC route. Protection of IV BALB/c immunized mice ranged between 12 and $75 \%$, but in four out of six experiments lesions were 40 to $75 \%$ 
smaller than control animals. Mice immunized with $10^{6} \mathrm{dhfr}-\mathrm{ts} \mathrm{s}^{-}$promastigotes displayed greater protection $(\mathrm{p}<0.05)$. Animals vaccinated with $10^{4}$ auxotrophic promastigotes showed in two experiments a worst course of infection with lesions 20 to $50 \%$ larger than to those from control animals (Fig. 1b).

The effect of two doses was compared to a single dose in both SC (Fig. 2) and IV (data not shown) routes of immunization. BALB/c mice were vaccinated with a SC dose of $10^{8}$ promastigotes, a dose, which conferred a higher degree of protection against $L$. amazonensis challenge. E10-5A3 promastigotes were inoculated three or one week or both three and one week before challenge. At five to six weeks after infection double-immunized animals displayed lesions $65 \%$

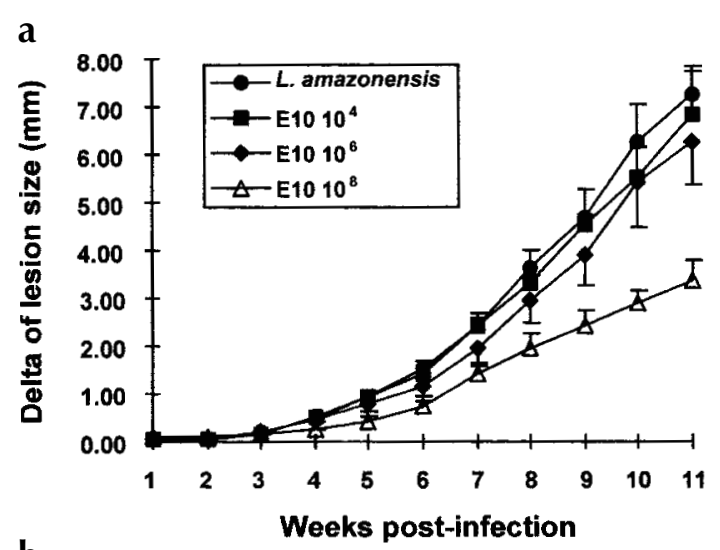

b

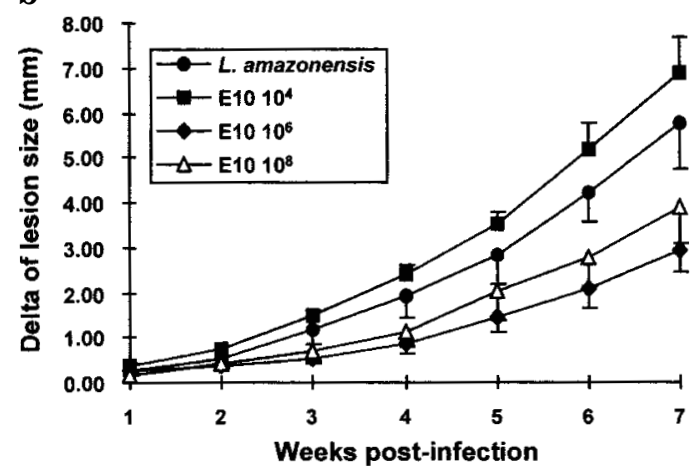

Fig. 1: E10-5A3 immunized BALB/c mice showed partial protection from Leishmania amazonensis challenge infection. Animals were vaccinated by (a) subcutaneous (SC) or (b) intravenous (IV) injection of $10^{4}, 10^{6}$ or $10^{8}$ stationary phase promastigotes of auxotrophic L. major and one week later, were challenged with $10^{6}$ (a) or $5 \times 10^{6}$ (b) stationary phase promastigotes of $L$. amazonensis in one hind footpad. Results represent the differences in lesion size of test animals contrasted with size of the contralateral footpad that received only saline instead of immunogen agent. Each point represents the mean of measurements done in five animals \pm SEM. The results shown are representative of four experiments for $\mathrm{SC}$ and seven experiments for IV immunization. smaller when compared to non-immunized animals $(p<0.001)$. Levels of protection in mice immunized once or twice doses were similar ( $p>0.05)$. In this experiment, compared to non immunized animals, lesions of those immunized three weeks before infection were 57 to $33 \%$ smaller $(\mathrm{p}<0.05)$ and of those immunized one week before infection were 42 to $45 \%$ ( $<<0.01)$ smaller (Fig. 2).

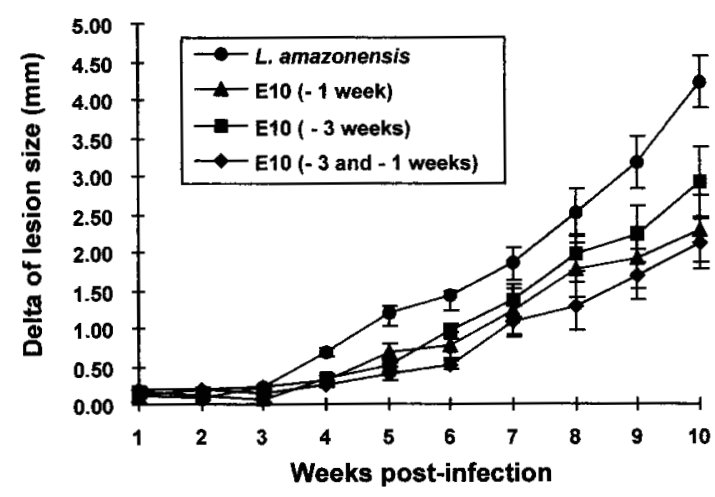

Fig. 2: BALB/C mice double-immunized with E10-5A3. Animals were vaccinated subcutaneously with $10^{8}$ stationary phase promastigotes of auxotrophic Leishmania major three weeks and again one week before L. amazonensis $\left(10^{6}\right)$ challenge infection. Each point represents the mean of measurements done in five animals \pm SEM. The results shown are similar to another experiment by IV immunization.

C57BL/6 more closely resembles human CL as they undergo clinical apparent cure after lesion development following $L$. amazonensis infection. Groups of C57BL/6 mice $(n=5)$ were vaccinated and challenged similarly to BALB/c mice. C57BL/ 6 mice immunized subcutaneously with $10^{8}$ E10$5 \mathrm{~A} 3$ promastigotes showed consistently higher protection against $L$. amazonensis infection compared to BALB/c mice. Subcutaneously immunized animals (Fig. 3a) displayed 50 to $65 \%$ protection compared to control unvaccinated mice $(\mathrm{p}<0.05)$. Intravenously immunized C57BL/6 mice showed protection ranging from 40 to $70 \%$ when compared to control mice $(\mathrm{p}<0.05)$. The $10^{6} \mathrm{IV}$ dose of auxotrophic promastigotes conferred the strongest protection of C57BL/6 mice against $L$. amazonensis infection (Fig. 3b). In general, the degree of protection obtained in these $L$. amazonensis partial resistant mice (Barral et al. 1983) was comparable or better than those obtained previously by homologous challenge with $L$. $m a$ jor (Titus et al. 1995). However, C57BL/6 mice were probably not sterilized and parasites did not were cleared at the site of infection, as immunization with E10-5A3 induced a partial protection against $L$. amazonensis. 


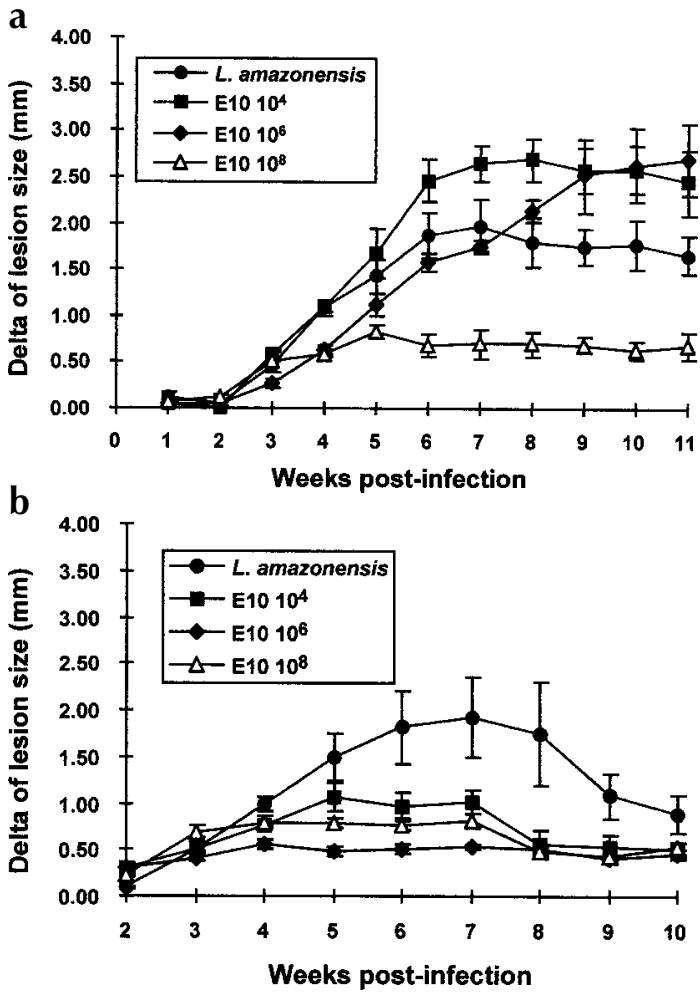

Fig. 3: E10-5A3 immunized C57BL/6 mice displayed partial protection from Leishmania amazonensis challenge infection. As in previous experiments, animals were vaccinated by (a) subcutaneous (SC) or (b) intravenous (IV) injection of $10^{4}, 10^{6}$ or $10^{8}$ stationary phase promastigotes of auxotrophic $L$. major and one week later, were challenged with $10^{6}$ (a) or $5 \times 10^{6}(\mathrm{~b})$ stationary phase promastigotes of L. amazonensis in one hind footpad. Results represent the differences in lesion size of test animals contrasted with size of the contralateral footpad that received only saline instead of immunogen agent. Each point shows the mean of measurements done in five animals \pm SEM. The results shown are representative of two experiments for $\mathrm{SC}$ and three experiments for IV immunization.

\section{DISCUSSION}

Large scale human trials have demonstrated that protective immunity can result from prior controlled induction of disease with a virulent parasite. These data suggested that infections with Leishmania species that produce benign self-healing lesions can be linked to a solid protective immunity (Barral-Netto \& Barral 1987, Modabber 1990, Grimaldi Jr 1995, Handman 1997). However, infection with virulent parasites in order to induce immunity is not an acceptable practice anymore, and workers have turned to the use of attenuated parasites instead (Rivier et al. 1993, Titus et al. 1995).

In this report we have studied a genetically defined attenuated $L$. major vaccine line, gener- ated by homologous gene targeting of the essential DHFR-TS locus. These $d h f r-t s^{-}$parasites are auxotrophic for thymidine, and incapable of causing disease in genetically susceptible mice (Titus et al. 1995). It has been previously demonstrated that vaccination with a $d h f r-t s^{-}$auxotrophic stationary phase or metacyclic promastigotes but not parasite lysate led to high levels of protection against homologous virulent $L$. major challenge infection, in both susceptible and resistant strains of mice (Titus et al. 1995). Although challenge was performed one week after E10-5A3 infection, we have demonstrated that the response was specific to parasites as lymphocytes proliferated only in response to mitogen or parasite stimuli and not to unrelated antigens (Titus et al. 1995). In this report we used the same vaccination protocol (Titus et al. 1995) and demonstrated that this auxotrophic mutant is also able to confer protection in both $\mathrm{BALB} / \mathrm{c}$ or $\mathrm{C} 57 \mathrm{BL} / 6$ mice against a heterologous challenge with $L$. amazonensis infection.

In our experiments the direct comparison of protection by SC and IV routes is slightly complicated as we used $10^{6} \mathrm{~L}$. amazonensis promastigotes to challenge mice immunized by SC route instead of $5 \times 10^{6}$ parasites to animals immunized intravenously. Protection can be achieved through SC immunization, but the level of protection found after IV immunization appears to be greater in the $10^{6}$ immunized animals. These results are encouraging as there are relatively few reports that demonstrate protection against Leishmania infection by SC vaccination. Non-viable L. major or L. amazonensis, when administered subcutaneously, showed no effect, although they were protective by the IV route (Howard et al. 1981, Barral et al. 1991). However, Rivier et al. (1993) demonstrated that radioattenuated $L$. major induced a protective SC immunization in resistant CBA mice against challenge infections by both homologous avirulent and heterologous virulent strains of $L$. major. Ideally, a vaccine against Leishmania would protect both susceptible and resistant individuals, when administered by SC or IM (intramuscular) inoculation routes (instead of IV or intraperitoneal routes).

An important consideration in vaccination is protection against challenge by heterologous strains or species of parasites. This is particularly important in South America, where many different strains and/or species of parasite occur close together. It is therefore encouraging that a reasonable degree of protection against $L$. amazonensis was obtained in our studies by vaccination with $L$. major. Together our results suggest that in human trials cross-protection could be achieved between parasites from Old and New Worlds (Alexander \& Phillips 1978, Alexander \& Blackwell 1983). Pos- 
sibly, a stronger degree of protection would be observed if a $d h f r$-ts ${ }^{-}$strain of L. amazonensis had been used as an immunogen. The degree of protection could also perhaps be enhanced by further genetic manipulations of this auxotrophic mutant or by the use of adjuvants (Afonso et al. 1994). Taken together our results indicate that the use of E10-5A3 or other attenuated parasites for protection against New World leishmaniasis is promising and deserves further study.

\section{ACKNOWLEDGMENTS}

To Dr Richard G Titus for helpful suggestions.

\section{REFERENCES}

Afonso LCC, Scharton TM, Vieira LQ, Wysocka M, Trinchieri G, Scott P 1994. The adjuvant effect of interleukin-12 in a vaccine against Leishmania major. Science 263: 235-237.

Alexander J, Blackwell JM 1986. The immunological significance of genetically determined cross reactivity between taxonomically distinct Leishmania species, p. 185-191. In Leishmania. Taxonomie et Phylogenèse. Applications éco-épidémiologiques, IMEEE, Montpellier.

Alexander J, Phillips RS 1978. Leishmania tropica and Leishmania mexicana: cross-immunity in mice. Exp Parasitol 45: 93-100.

Barral A, Pedral SD, Grimaldi Jr G, Momen H, MacMahon PD, Ribeiro DJA, Almeida R, Badaró R, Barral-Netto M, Carvalho EM, Johnson WJ 1991. Leishmaniasis in Bahia, Brazil: evidence that Leishmania amazonensis produces a wide spectrum of clinical disease. Am J Trop Med Hyg 44: 536-546.

Barral A, Petersen EA, Sacks DL, Neva FA 1983. Late metastatic leishmaniasis in mouse. A model for mucocutaneous disease. Am J Trop Med Hyg 32: 277285.

Barral-Netto M, Barral A 1987. Imunização específica nas leishmanioses tegumentares. Mem Inst Oswaldo Cruz 82: 293-302.

Belosevic M, Finbloom DS, Van der Meide PH, Slayter MV, Nacy CA 1989. Administration of monoclonal anti-IFN- $\gamma$ antibodies in vivo abrogates natural resistance of $\mathrm{C} 3 \mathrm{H} / \mathrm{HeN}$ mice to infection with Leishmania major. J Immunol 143: 266-274.

Bogdan C, Gessner A, Solbach W, Rolinghoff M 1996. Invasion, control and persistence of leishmania parasites. Cur Opinion Immunol 8: 517-625.

Carvalho EM, Badaró R, Reed SG, Jones TC, Johnson WJ 1985a. Absence of gamma interferon and interleukin 2 production during active visceral leishmaniasis. J Clin Invest 76: 2066-2069.

Carvalho EM, Johnson WD, Barreto E, Marsden PD, Costa JLM, Reed SG, Rocha H 1985b. Cell mediated immunity in American cutaneous and mucosal leishmaniasis. J Immunol 135: 4144-4148.

Connell ND, Medina-Acosta E, McMaster WR, Bloom BR, Russell DG 1993. Effective immunization against cutaneous leishmaniasis with recombinant bacille Calmette-Guérin expressing the leishmania surface proteinase gp63. Proc Nat Acad Sci USA 90: 11473-11477.

Cruz A, Beverley SM 1990. Gene replacement in parasitic protozoa. Nature 348: 171-173.

Cruz A, Coburn CM, Beverley SM 1991. Double targeted gene replacement for creating null mutants. Proc Nat Acad Sci USA 88: 7170-7174.

Desjeux P 1992. Human leishmaniasis: epidemiology and public health aspects. World Health Statis Quant 45: 267-275.

Grimaldi Jr G 1995. Meeting on vaccine studies towards the control of leishmaniasis. Mem Inst Oswaldo Cruz. 90: 553-556.

Handman E 1997. Leishmania vaccines: old and new. Parasitol Today 13: 236-238.

Handman E, Hocking RE, Mitchell GF, Spithill TW 1983. Isolation and characterization of infective and non-infective clones of Leishmania tropica. Mol Biochem Parasitol 7: 111-126.

Howard JG, Hale C, Liew FY 1981. Immunological regulation of experimental cutaneous leishmaniasis. IV. Prophylactic effect of sublethal irradiation as a result of abrogation of suppressor $\mathrm{T}$ cell generation in mice genetically susceptible to Leishmania tropica. $J$ Exp Med 153: 557-568.

Howard JG, Nicklin S, Hale C, Liew FY 1982. Prophylactic immunization against experimental leishmaniasis: I. Protection induced in mice genetically vulnerable to fatal Leishmania tropica infection. $J$ Immunol 132: 450-455.

Kimsey PB, Theodos CM, Mitchen TK, Turco SJ, Titus RG 1993. An avirulent lipophosphoglycan-deficient Leishmania major clone induces $\mathrm{CD} 4^{+} \mathrm{T}$ cells which protect susceptible BALB/c mice against infection with virulent $L$. major. Inf Immun 61: 5205-5213.

Lainson R, Shaw JJ 1977. Leishmaniasis in Brazil. XII. Observations on cross-immunity in monkeys and man infected with Leishmania mexicana mexicana, L. m. amazonensis, L. braziliensis braziliensis, $L$. $b$. guyanensis and L. b. panamensis. J Trop Med Hyg 80: 29-35.

Launois P, Maillard I, Pingel S, Swithart KG, Xénarios I, Acha-Orbea H, Diggelmann H, Locksley RM, MacDonald HR, Louis JA 1997. IL-4 rapidly produced by $\mathrm{V} \beta 4 \mathrm{~V} \alpha 8 \mathrm{CD} 4^{+} \mathrm{T}$ cells intructs $\mathrm{Th} 2$ development and susceptibility to Leishmania major in BALB/c mice. Immunity 6: 541-549.

Marchand M, Dadout S, Titus RG, Louis J, Boon T 1987. Variants with reduced virulence derived from Leishmania major after mutagen treatment. Par Immunol 9: 81-92.

Mitchell GF, Handman E, Spithill TW 1984. Vaccination against cutaneous leishmaniasis in mice using nonpathogenic cloned promastigotes of Leishmania major and importance of route of injection. Aust $J$ Exp Biol Med Sci 62: 145-153.

Modabber F 1990. Development of vaccines against leishmaniasis. Scand J Infect Diseases 76 (Supp.): 72-78.

Romagnani S 1996. Understanding the role of Th1/Th2 cells in infection. Trends Microbiol 4: 470-473.

Rivier D, Shah R, Bovay P, Mauel J 1993. Vaccine de- 
velopment against cutaneous leishmaniasis. Subcutaneous administration of radioattenuated parasites protects CBA mice against virulent Leishmania major challenge. Par Immunol 15: 75-84.
Titus RG, Gueiros-Filho F, de Freitas LAR, Beverley SM 1995. Development of a safe live leishmania vaccine line by gene replacement. Proc Nat Acad Sci USA 92: 10267-10271. 